\title{
Design and rationale of a randomized controlled trial of melatonin supplementation in men and women with the metabolic syndrome
}

This article was published in the following Dove Press journal:

Open Access Journal of Clinical Trials

5 March 2013

Number of times this article has been viewed

Paul D Terry'

Abhinav Goyal2,3

Lawrence S Phillips ${ }^{3}$

Hillary M Superak ${ }^{4}$

Michael H Kutner ${ }^{4}$

'Departments of Public Health and Surgery, University of Tennessee, Knoxville, TN, ${ }^{2}$ Department of Epidemiology, Emory Rollins School of Public Health, ${ }^{3}$ Department of Medicine, Emory School of Medicine, ${ }^{4}$ Department of Biostatistics and Bioinformatics, Emory Rollins School of Public Health, Atlanta, GA, USA
Correspondence: Michael H Kutner Department of Biostatistics and Bioinformatics, Emory Rollins School of Public Health, 1518 Clifton Road NE, Atlanta, GA 30322-420I, USA

$\mathrm{Tel}+\mathrm{I} 4047129708$

Fax + I 404727 1370

Email mkutner@emory.edu
Background: The metabolic syndrome is a constellation of interrelated metabolic risk factors that appear to increase the risk of atherosclerotic cardiovascular disease, type 2 diabetes mellitus, and possibly some cancers. Animal studies and observational clinical data in humans suggest that supplemental melatonin may ameliorate a number of components of the metabolic syndrome, including elevated glucose, elevated blood pressure, dyslipidemia, and obesity. The primary objective of this clinical trial was to determine the feasibility, efficacy, and safety of melatonin supplementation in men and women with the metabolic syndrome.

Methods: Thirty-nine men and women of mixed race/ethnicity were enrolled into a randomized, double-blind, placebo-controlled clinical trial with two arms: placebo for 10 weeks followed by melatonin for 10 weeks, or vice versa, with an interval 6-week washout period, in a crossover trial design. Outcome measures include metabolic syndrome components (blood pressure, glucose, triglycerides, high-density lipoprotein cholesterol, waist circumference), oxidative stress, and inflammation biomarkers. These biomarkers, along with sleep duration and quality and pretreatment endogenous melatonin levels, were measured to explore possible underlying biologic mechanisms.

Discussion: This trial will provide knowledge of the effects of melatonin in metabolic syndrome subjects, and lay the groundwork for future clinical trials of melatonin in metabolic syndrome subjects.

Keywords: melatonin, metabolic syndrome, diabetes, blood pressure, sleep

\section{Introduction}

The metabolic syndrome is a constellation of interrelated metabolic risk factors that appear to increase the risk of atherosclerotic cardiovascular disease, type 2 diabetes mellitus, and possibly some cancers. ${ }^{1-3}$ Although pharmacologic treatments for some of the metabolic syndrome components exist, these treatments are not always effective and are often associated with adverse effects. Therefore, there is considerable interest in finding alternative treatments for the metabolic syndrome.

Melatonin (N-acetyl-5-methoxytryptamine) is an endogenous indoleamine compound (chemical formula $\mathrm{C}_{13} \mathrm{H}_{16} \mathrm{~N}_{2} \mathrm{O}_{2}$ ). Melatonin has chronobiotic, antioxidant, antiinflammatory, and soporific properties. A major site of melatonin biosynthesis is the pineal gland, from which melatonin is released into the circulation in response to photic light/dark information transmitted from the retina to the suprachiasmatic nucleus of the hypothalamus. ${ }^{4}$ Pineal melatonin production and release follows a circadian pattern, with increased release in darkness and decreased release during exposure to light. It is understood that one of the main physiologic functions of melatonin is to transmit 
information about the light/dark cycle to the body, thereby allowing the body's physiologic systems to orient themselves according to seasonal and circadian patterns. ${ }^{4}$

Animal studies and observational clinical data in humans suggest that supplemental melatonin may ameliorate a number of metabolic syndrome components, including elevated glucose, elevated blood pressure, dyslipidemia, and obesity. ${ }^{5-9}$ The evidence also suggests that individuals with metabolic syndrome components have suppressed melatonin levels, and hence may be particularly likely to benefit from exogenous melatonin supplementation. ${ }^{10}$ On the whole, evidence from animal experiments and observational epidemiologic studies suggest that endogenous melatonin or melatonin supplementation may protect against elevated glucose/insulin resistance, elevated blood pressure, dyslipidemia, and perhaps abdominal obesity. However, the evidence is insufficient in several ways, including the lack of randomized clinical trials in humans, particularly among individuals with the metabolic syndrome. ${ }^{9}$

The purpose of this paper is to present the design, rationale, and methodologic considerations of a randomized, double-blinded, placebo-controlled, clinical trial of melatonin supplementation in men and women with the metabolic syndrome. This trial will assess the feasibility of recruitment of metabolic syndrome subjects and will determine if treatment with melatonin (10 weeks) can ameliorate one or more of the metabolic syndrome components, ie, blood pressure, glucose, triglycerides, high-density lipoprotein (HDL) cholesterol, and waist circumference. Our long-term goal is to determine the safety and efficacy of melatonin to reduce the risk of development of type 2 diabetes and cardiovascular disease in high-risk individuals with the metabolic syndrome.

\section{Materials and methods Overview}

The Melatonin and the Metabolic Syndrome (MetSyn) study was a randomized, double-blind, placebo-controlled clinical trial with two arms, ie, placebo for 10 weeks followed by melatonin for 10 weeks or vice versa, with an interval 6-week washout period, in a crossover trial design. ${ }^{11}$ Thirtynine subjects were randomized to one of the following two treatments, ie, placebo once daily one hour before bedtime for 10 weeks or $8.0 \mathrm{mg}$ oral melatonin supplementation once daily one hour before bedtime for 10 weeks. At the end of the first 10-week period, each subject underwent a 6-week washout period in which no pills were taken. At the end of the washout period, the subjects were then crossed over to the alternative treatment for 10 weeks. Using this crossover design, each subject serves as his or her own control. Recruitment, randomization, and subject follow-up have been completed (Figure 1), and laboratory assays and data cleaning are nearly complete.

Subjects in the MetSyn study were recruited from several sources: the population of an already completed study funded by the National Institute of Diabetes and Digestive and Kidney Diseases of the National Institutes of Health, ie, Screening for IGT: Glucose Challenge versus Predictive Model (SIGT, R18DK066204; Lawrence S Phillips, principal investigator); the Preventive Cardiology Clinic at the Emory Clinic in Atlanta, GA, USA; and advertisements placed on the campus of the Emory Clinic. The purpose of the SIGT study was to test the hypothesis that screening by a one-hour oral glucose challenge test would have good predictive ability to identify impaired glucose tolerance, and would be superior to conventional predictive models in both diagnostic efficiency and cost-effectiveness. SIGT enrolled a sample of 525 metabolic syndrome subjects who were Emory University employees.

The MetSyn study enrolled a total of 39 men and women (aged 30-79 years) who met the criteria for metabolic syndrome (described below). Subject visits for the clinical trial were at the Emory University General Clinical Research Center, also known as the Atlanta Clinical and Translational Science Institute. Subjects were compensated financially for their participation in the trial.

\section{Inclusion and exclusion criteria}

Consistent with the criteria established by the National Heart, Lung, and Blood Institute and the American Heart Association, ${ }^{1}$ an individual was defined as having the metabolic syndrome if he/she had three or more of the following five conditions: elevated fasting glucose ( $\geq 100 \mathrm{mg} / \mathrm{dL})$, enlarged blood pressure $(>130 \mathrm{mmHg}$ systolic or $>85 \mathrm{mmHg}$ diastolic), elevated triglycerides ( $\geq 150 \mathrm{mg} / \mathrm{dL}$ ), reduced HDL cholesterol ( $<40 \mathrm{mg} / \mathrm{dL}$ in men, $<50 \mathrm{mg} / \mathrm{dL}$ in women), and enlarged waist circumference ( $\geq 102 \mathrm{~cm}$ in men, $\geq 88 \mathrm{~cm}$ in women). Subjects previously diagnosed with diabetes mellitus were excluded. To avoid the possibility of an increased risk of developmental disorders, we excluded women who were pregnant, breast-feeding, or attempting or planning to attempt conception. We also excluded men and women with the following medical conditions: active malignancy (other than nonmelanoma skin cancer); hyperthyroidism or hypothyroidism; heart failure (New York Heart Association functional class III or IV); history of myocardial infarction, bypass surgery, angioplasty, or stroke within the past year; active liver disease; active pancreatitis or pancreatic insufficiency; active peptic 


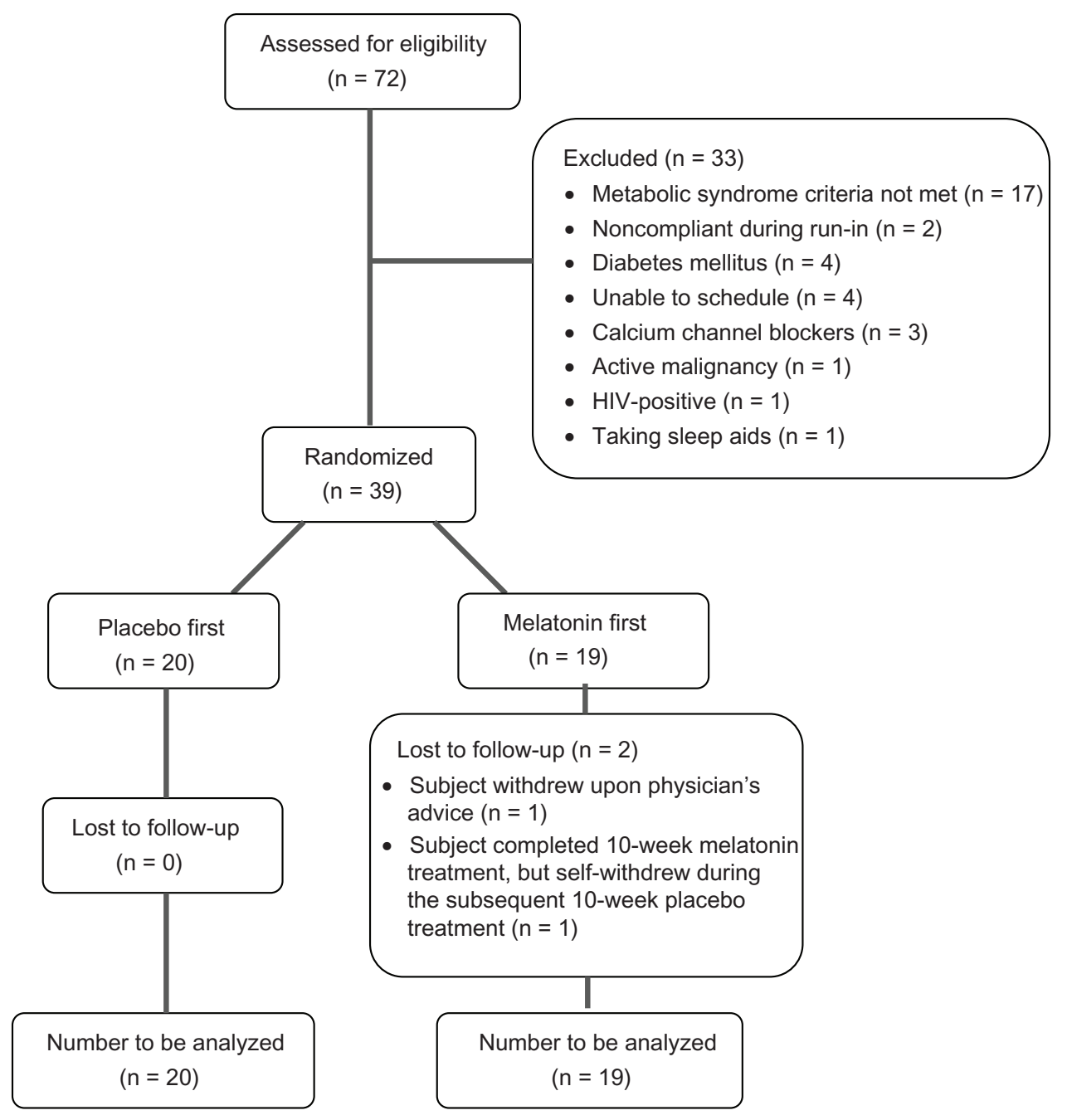

Figure I Subject recruitment flow chart.

ulcer disease; previous gastrectomy or bowel resection; intestinal malabsorption syndrome; narcotic or alcohol dependence; recent significant $(\geq 10 \%)$ intentional or unintentional weight loss or weight gain; end-stage kidney disease on dialysis; taking immunosuppressive therapy (eg, systemic corticosteroids, azathioprine, methotrexate, cyclophosphamide); taking lithium therapy; or any condition with a life expectancy of less than 2 years. We also excluded subjects taking calcium channel blockers because a clinical study of melatonin and blood pressure suggested that melatonin supplementation may have increased blood pressure in persons taking calcium channel blockers. ${ }^{12}$ Finally, we limited enrollment to nonsmokers based on evidence of potential confounding from tobacco smoking. ${ }^{10}$

\section{Recruitment and screening}

We screened men and women who appeared to meet eligibility criteria, received support for participation from their physicians, and expressed interest in the study. After informed consent, we confirmed further eligibility after reviewing medical charts, and conducting in-person interviews, a medical examination, and screening blood tests. If the subject was eligible after all these steps and still willing to participate, the subject underwent a 10-day placebo run-in during which time adherence to therapy was assessed. Even though only placebo was administered during the run-in phase, subjects were informed only that they were taking "study drug." Subjects who had taken at least $80 \%$ of their tablets (placebo) without bothersome side effects were randomized.

\section{Randomization}

Randomization was performed independently by a biostatistician not involved in the study. Four randomly generated permuted blocks of size 10 (five placebo and five melatonin) were generated. The order of randomization 
for the estimated 40 subjects was placed in sealed envelopes and given to the clinical pharmacy department. The pharmacy used these envelopes to determine the randomization status for each subject in the study. Subjects were initially randomized to one of the following two treatments: placebo once daily one hour before bedtime for 10 weeks or $8.0 \mathrm{mg}$ melatonin once daily one hour before bedtime for 10 weeks. At the end of the first 10-week period, each subject underwent a 6-week washout period in which no pills were taken. At the end of the washout period, the subject was crossed over to the alternative treatment for 10 weeks (Figure 2).

\section{Enrolled participants}

During the study, 72 subjects were screened for participation, 33 were excluded, and 39 were randomized (Figure 1). The demographic and metabolic baseline characteristics of the 39 enrolled subjects are shown (Table 2). The mean age at enrollment was 58.9 years. Over half of the subjects were women, and 33\% were African-American.

\section{Treatment handling, dosage, duration, rationale, toxicity, and safety}

Both the study drug and placebo were supplied by the Investigational Drug Service of Emory University. Melatonin powder was obtained from Professional Compounding Centers of America, which obtains melatonin from Marcor Development Corporation. Certificates of purity were provided for each batch of melatonin. Placebo capsules were compounded from lactose powder and were identical in appearance to the melatonin capsules. All compounding was completed by the Investigational Drug Service of Emory University Hospital, which stored the drug and placebo and dispensed prescriptions to subjects enrolled in the study. The project manager gave the bottles of pills to the subjects at each visit.

Random samples of the melatonin lot were sent to consumerlab.com for independent verification. Consumerlab. com uses high-performance liquid chromatography and inductively coupled plasma mass spectrometry analytic methods to verify the biochemical composition of various drugs/supplements, and has extensive experience testing melatonin preparations.

In this trial, subjects were instructed to take melatonin one hour before bedtime, typically close to $9 \mathrm{pm}$. The rationale for administering melatonin before bedtime is that it prevents the soporific effects of melatonin from interfering with the subject's usual activities during the day, and other trials have typically administered melatonin around bedtime, facilitating the comparison of study results.

The doses of melatonin used in human clinical trials has ranged from $0.3 \mathrm{mg} /$ day to $10.0 \mathrm{mg} /$ day. ${ }^{9,13,14}$ The duration of melatonin clinical trials has ranged from one-time administration, including blood pressure and sleep trials, to daily dosing for up to 12 months. ${ }^{14}$ However, the typical duration is $1-4$ weeks. The two most significant concerns regarding melatonin supplementation are: causing sleepiness in some people with daytime doses of $8.0 \mathrm{mg} /$ day, and giving melatonin at a time when it might cause an unwanted sleep/ wake phase shift. The list of side effects that we monitored included early morning wakening (a possible sign of a small phase advance), headaches, dizziness, nausea, and drowsiness. ${ }^{14}$ In a meta-analysis addressing the efficacy and safety of exogenous melatonin in 487 subjects, the distribution of these potential side effects was similar between the melatonin and placebo groups for doses up to $7.5 \mathrm{mg}$ /day and for durations up to 12 months. ${ }^{14}$ Based on these prior studies, we deemed an $8.0 \mathrm{mg}$ daily dose to be safe and tolerable, and a 10-week

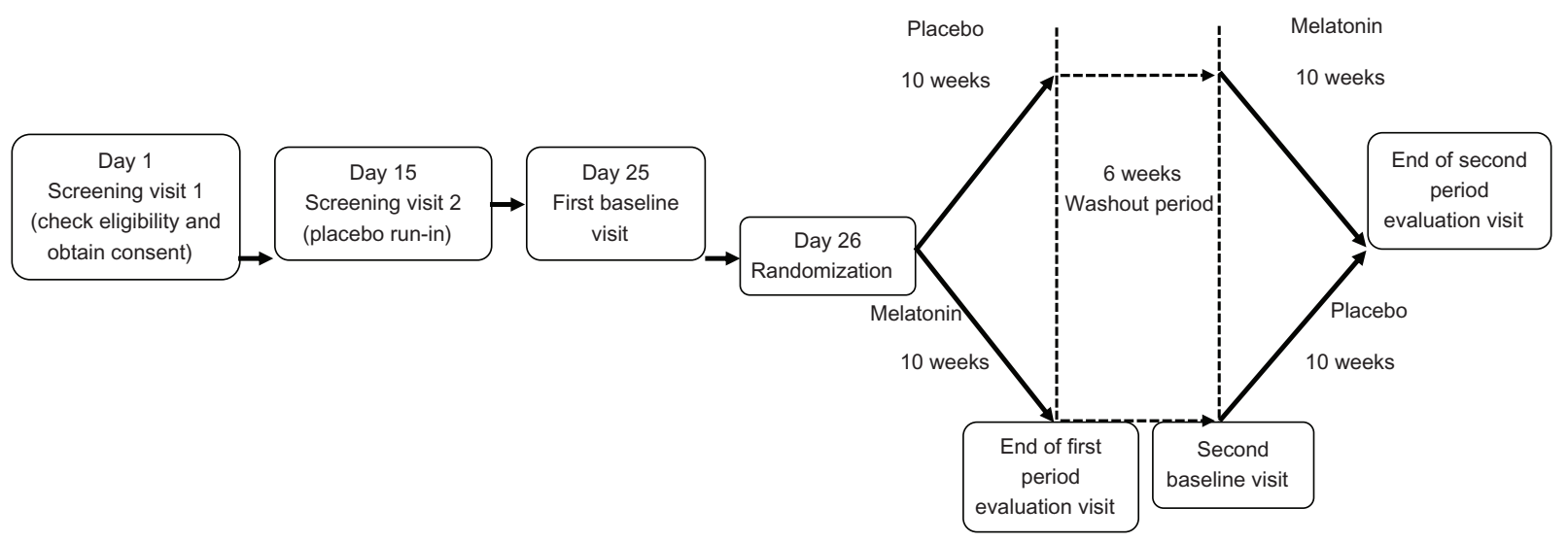

Figure 2 Schematic of study design. 
Table I Power to detect within-subject differences in components of the metabolic syndrome after treatment with melatonin compared with placebo using a crossover design, assuming that 30 subjects completed the trial

\begin{tabular}{|c|c|c|c|c|}
\hline $\begin{array}{l}\text { Metabolic } \\
\text { syndrome } \\
\text { component }\end{array}$ & $\begin{array}{l}\text { Projected mean within- } \\
\text { subject difference } \\
\text { (melatonin - placebo) }\end{array}$ & $\begin{array}{l}\text { Projected within- } \\
\text { subject standard } \\
\text { deviation }\end{array}$ & $\begin{array}{l}\text { Power to detect projected } \\
\text { mean within subject- } \\
\text { difference (melatonin - } \\
\text { placebo): } \alpha=0.05\end{array}$ & $\begin{array}{l}\text { Power to detect projected } \\
\text { mean within subject- } \\
\text { difference (melatonin - } \\
\text { placebo): } \alpha=0.0 \text { I }\end{array}$ \\
\hline $\begin{array}{l}\text { Fasting glucose } \\
(\mathrm{mg} / \mathrm{dL})\end{array}$ & -5.5 & 6.7 & 0.86 & 0.66 \\
\hline $\begin{array}{l}\text { 24-hour systolic blood } \\
\text { pressure }(\mathrm{mmHg})\end{array}$ & -5.5 & 7.4 & 0.79 & 0.55 \\
\hline $\begin{array}{l}\text { HDL cholesterol } \\
(\mathrm{mg} / \mathrm{dL})\end{array}$ & +3.6 & 4.7 & 0.82 & 0.59 \\
\hline Triglycerides (mg/dL) & -7.5 & 10.0 & 0.80 & 0.56 \\
\hline $\begin{array}{l}\text { Waist } \\
\text { circumference }(\mathrm{cm})\end{array}$ & -4.0 & 5.4 & 0.79 & 0.55 \\
\hline
\end{tabular}

Abbreviation: HDL, high-density lipoprotein cholesterol.

period to be a sufficient duration of time for the components of the metabolic syndrome to be influenced.

\section{Retention and compliance}

At the initial interview visit, all potential subjects received an orientation to the study. Spouses and housemates were encouraged to attend this orientation because they could corroborate whether or not the study subjects took their medications as prescribed. An information booklet described how and when to take the study pills and what to do if problems were experienced (eg, lost pills or side effects). The project manager also provided participants with a subject log so that they could document any unusual symptoms while they were taking the study medication. Participants were also provided with the addresses (with postcards) and phone numbers of the project investigators and project manager. The project manager also educated each subject about how and where to store the study pills and what time of day to take them.

\section{Study outcomes}

The primary outcomes were levels of the following factors after 10 weeks of melatonin or placebo supplementation: plasma glucose, office and 24-hour ambulatory blood pressure values, triglyceride levels, HDL cholesterol levels, and waist circumference. These levels were measured on four occasions during the study, ie, at the start and end of the first 10-week period, and then (following the 6-week washout period) again at the start and end of the second 10-week period (Figure 2).

\section{Fasting glucose}

Glucose was measured by the Emory University Hospital Core Laboratory from fresh plasma samples within 6 hours after collection. Prior to sample collection, participants were advised to fast overnight and to not eat breakfast. GLUCm reagent is used in conjunction with the Synchron LX system and Synchron LX Aqua Cal 1 and 2 (Beckman Coulter, Fullerton, CA, USA) to measure quantitative glucose levels.

\section{Blood pressure}

We measure 24-hour ambulatory blood pressure using a Spacelabs 90217 ambulatory blood pressure monitor. This monitor has been validated for the measurement of systolic and diastolic blood pressure. ${ }^{15}$ The monitor is programmed to record blood pressure every 60 minutes for a 24 -hour period. In addition, during General Clinical Research Center visits at the beginning and end of each of the 10-week treatment periods, blood pressure was measured using a sphygmomanometer 5 minutes after the participant had been seated, twice in each of the right and left arms.

\section{Triglycerides}

Triglycerides were measured by the Emory University Hospital Core Laboratory from fresh plasma within 6 hours of collection. Triglycerides glycerophosphate oxidase reagent is used in conjunction with the Synchron LX System, the Synchron systems multicalibrator, and triglycerides-blanked assay parameters (Beckman Coulter) to determine quantitative triglyceride levels. Triglycerides glycerophosphate oxidase reagent is used to measure the triglycerides concentration by a timed endpoint method. Triglycerides in the sample are hydrolyzed to glycerol and free fatty acids by the action of lipase. A sequence of three coupled enzymatic steps using glycerol kinase, glycerophosphate oxidase, and horseradish peroxidase causes the oxidative coupling of 3,5-dichloro-2hydroxybenzenesulfonic acid with 4-aminoantipyrine to form 
Table 2 Baseline characteristics of enrolled subjects $(n=39)$

\begin{tabular}{|c|c|c|c|c|}
\hline Characteristic & $\begin{array}{l}\text { Mean } \pm \text { SD or } \\
\text { n (\%) }\end{array}$ & Median & Minimum & Maximum \\
\hline Age (years) & $60.0 \pm 10.1$ & 59.4 & 35.0 & 78.1 \\
\hline \multicolumn{5}{|l|}{ Gender } \\
\hline Male & 17 (43.6\%) & - & - & - \\
\hline Female & $22(56.4 \%)$ & & & \\
\hline \multicolumn{5}{|l|}{ Race } \\
\hline Caucasian & $24(61.5 \%)$ & - & - & - \\
\hline African American & $13(33.3 \%)$ & & & \\
\hline Asian & $\mathrm{I}(2.6 \%)$ & & & \\
\hline Hispanic/Latino & I (2.6\%) & & & \\
\hline \multicolumn{5}{|l|}{ Waist circumference $(\mathrm{cm})$} \\
\hline Males & $112.5 \pm 8.9$ & 111.3 & 99.1 & 133.1 \\
\hline Females & $103.9 \pm 10.2$ & 102.9 & 88.4 & 132.1 \\
\hline Triglycerides (mg/dL) & $181.8 \pm 168.6$ & 140.1 & 55.0 & 1043.7 \\
\hline \multicolumn{5}{|l|}{ HDL cholesterol (mg/dL) } \\
\hline Males & $36.7 \pm 6.4$ & 34.4 & 28.1 & 50.2 \\
\hline Females & $46.8 \pm 10.2$ & 44.4 & 32.1 & 74.0 \\
\hline \multicolumn{5}{|l|}{ Blood pressure $(\mathrm{mmHg})^{*}$} \\
\hline Systolic & $126.1 \pm 16.9$ & 123.5 & 89.5 & 161.3 \\
\hline Diastolic & $77.1 \pm 11.3$ & 74.0 & 57.5 & 103.8 \\
\hline Fasting glucose $(\mathrm{mg} / \mathrm{dL})$ & $102.6 \pm 13.8$ & 102.8 & 74.0 & 137.9 \\
\hline
\end{tabular}

Note: *Calculated as the average of four measurements (two per arm).

Abbreviation: HDL, high-density lipoprotein cholesterol.

a red quinoneimine dye. The Synchron LX system monitors the change in absorbance at $520 \mathrm{~nm}$ just prior to addition of lipase and for a fixed time interval after lipase addition. This change in absorbance is directly proportional to the concentration of triglycerides in the sample and is used by the system to calculate and express the triglyceride concentration.

\section{HDL cholesterol}

HDL cholesterol is measured by the Emory University Hospital Core Laboratory from fresh plasma samples within 6 hours of collection. HDLD reagent is used in conjunction with the Synchron LX system and Synchron systems lipid calibrator (Beckman Coulter) to determine quantitative HDL levels. HDLD reagent is used to measure cholesterol concentrations by a timed-endpoint method. The Synchron LX system automatically proportions the appropriate HDL cholesterol sample and reagent volumes into a cuvette. The ratio used is one part sample to 93 parts reagent. The system monitors the change in absorbance at $560 \mathrm{~nm}$, which is directly proportional to the concentration of cholesterol in the sample and is used by the system to calculate and express the HDL cholesterol concentration.

\section{Waist circumference}

We measured waist circumference using the procedure employed by the 2003-2004 wave of the National Health and
Nutrition Examination Survey. ${ }^{16}$ Briefly, this procedure involves locating the lateral border of the ilium of the standing study subject, drawing a horizontal line just above the uppermost lateral border of the right ilium, placing a measuring tape around the trunk in a horizontal plane at the level marked on the right side of the ilium, and measuring at the end of a normal expiration. Measurements are recorded to the nearest $0.1 \mathrm{~cm}$.

\section{Other study variables}

\section{Interview data}

The project manager interviewed subjects at the baseline visit to obtain data on demographics, health history, medication use, and dietary and behavioral factors. A questionnaire was administered at the end of each 10 -week period to assess potential side effects of exposure to melatonin or placebo. We asked specific questions regarding subject experiences of nausea, dizziness, fatigue, daytime drowsiness, early morning waking, and any other sleep disturbance, as well as any other unusual symptoms the participant may have experienced during the trial.

\section{Endogenous melatonin}

Endogenous melatonin levels in overnight urine (first morning void) samples will be measured using a radioimmunoassay (Alpco Diagnostic, Windham, NH, USA) with a sensitivity of $0.2 \mathrm{pg} / \mathrm{mL}$. Differences in morning melatonin levels are 
representative of relative differences in night-time and overall melatonin levels. ${ }^{17}$ Analyses will be performed to examine whether the effects of melatonin supplementation are modified by endogenous melatonin level.

\section{Sleep duration and quality}

We assessed sleep duration and quality using two approaches: a standard sleep log used by the Emory Program in Sleep, Aging, and Chronobiology ${ }^{18}$ and a conventional questionnaire to measure sleep quality, ie, the Pittsburgh Sleep Quality Index. ${ }^{19}$ The sleep log collected daily data on total sleep time. The Pittsburgh Sleep Quality Index, which has a one-month time frame, was also to be administered at the screening visit and three subsequent study visits, including the final visit. Analyses were performed to examine whether the effects of melatonin supplementation may be acting in part through changes in sleep duration and/or quality.

\section{Oxidative stress and inflammation biomarkers}

To assess potential underlying biologic mechanisms, we are assessing blood levels of several key markers of inflammation and oxidative stress, including high-sensitive $\mathrm{C}$-reactive protein, tumor necrosis factor-alpha, interleukin-6, F2-isoprostanes, and oxidized low-density lipoprotein cholesterol.

\section{Sample size and power calculations}

We conducted statistical power calculations to assess the ability of our trial to detect moderate, clinically meaningful improvements in our primary endpoints in this Phase II crossover trial. We planned to recruit 40 subjects with at least 30 of them completing the trial. Therefore, we calculated power for a sample of 30 subjects (Table 1). To estimate standard deviations, we used values of the between-subject standard deviations for each of our primary endpoints as reported in previous studies of metabolic syndrome. ${ }^{20-22}$ We assumed conservatively that the standard deviations in our study would be $66 \%$ of the between-subject standard deviations from these previous studies. For triglycerides, we based our estimates on the likely variability on the natural log-transformed values because these data are typically rightskewed. After making these assumptions, we found that we would have approximately $80 \%$ statistical power to detect each moderate effect size using a two-sided type I error rate (alpha) of $5 \%$ based on 30 subjects who completed the crossover design (Table 1). If we use a Bonferroni adjustment alpha of $0.05 / 5=0.01$, then the statistical power is reduced to approximately $55 \%-65 \%$ for each of the five comparisons. We also calculated the statistical power for the "global" test of whether melatonin improves at least one of the five metabolic syndrome endpoints. The power calculations were based on the multivariate Hotelling $\mathrm{T}^{2}$ module in PASS 2005 (Kaysville, UT, USA). Based on a sample size of 30 subjects, we achieved statistical power exceeding $90 \%$ for the global test, with a two-sided alpha of 0.05 .

\section{Data management}

Microsoft Access, an American National Standards Institute compliant relational database management system, was used for data entry and management. The data entry screens mimic paper case report forms for efficient data entry. Double data entry was utilized.

\section{Data analysis plan}

Analysis of primary endpoints was performed according to the intent-to-treat principle. Standard statistical methods for a one-period crossover design were employed using Statistical Analysis Software (SAS Inc, Cary, NC, USA). This includes testing for both a carryover effect and an order effect. To assess the possibility that subjects were aware of their treatment order, we collected data at the end of treatment on whether each subject thought he or she was first randomized to take melatonin or placebo. Dropouts were small in number and not deemed to be related to the study treatment. Specifically, two subjects dropped out during follow-up: one male participant dropped out upon his physician's advice in order to receive calcium channel blocking medication, and another male participant self-withdrew (without explanation) during the second of the two 10-week treatments, when the participant was taking placebo. These two subjects with partial data are included in the intent-to-treat data analysis of the primary and secondary endpoints.

\section{Discussion}

Melatonin supplements are widely used in the US, with many millions of dollars in annual sales. Understanding the effects of this widely used supplement on metabolic conditions that affect many individuals is an important public health issue.

Although most of the literature suggests that melatonin is safe and not associated with any major side effects, ${ }^{14}$ few studies have examined the effect of melatonin supplementation for the duration that we have planned. Although studies that have conducted long-term trials and/or with higher doses of melatonin have not reported serious side effects, subjects in our study were monitored carefully for any potential adverse effects.

We chose a crossover design for the MetSyn trial rather than a parallel group design in order to maximize efficiency. ${ }^{11}$ We assumed that neither a carryover effect nor an 
order-of-treatment effect was likely, nor was a significant loss to follow-up over the 26-week period of the trial. As described above, a placebo run-in period of ten days prior to randomization (to assess compliance) and a 6-week washout period between the first treatment period and the second treatment period (to eliminate a possible carryover effect) were key design features of this trial.

On the whole, evidence from animal experiments, observational epidemiologic studies, and a previous clinical trial suggest that melatonin supplementation may protect against elevated glucose/insulin resistance, elevated blood pressure, dyslipidemia, and perhaps abdominal obesity. ${ }^{5-9}$ However, the evidence is limited in part due to the lack of randomized clinical trials in humans. ${ }^{9}$ There have been few clinical trials of melatonin supplementation to assess effects on glucose levels, blood lipid levels, or body weight and/or size. Although there have been more clinical trials of melatonin supplementation focused on blood pressure, most have been of small sample size and their results have been inconsistent. This trial will provide knowledge of the effects of melatonin in subjects with metabolic syndrome, and lay the groundwork for future clinical trials of melatonin in subjects with metabolic syndrome.

\section{Acknowledgment}

This trial is approved by the Emory University institutional review board (14784), has Investigational New Drug approval by the US Food and Drug Administration (105764), and is registered at ClinicalTrials.Gov (01038921). The data safety monitoring board comprised William McClellan, Sol Jacobs, and Janice Lea, Emory University School of Medicine. The study was funded by the National Institutes of Health/National Center for Complementary and Alternative Medicine R21 AT004220-01A2, and was supported by the National Center for Advancing Translational Sciences of the National Institutes of Health under Award Number UL1TR000454. The content is solely the responsibility of the authors and does not necessarily represent the official views of the National Institutes of Health.

\section{Disclosure}

The authors report no conflicts of interest in this work.

\section{References}

1. Grundy SM, Brewer HB Jr, Cleeman JI, Smith SC Jr, Lenfant C; American Heart Association, National Heart, Lung, and Blood Institute. Definition of metabolic syndrome: Report of the National Heart, Lung, and Blood Institute/American Heart Association conference on scientific issues related to definition. Circulation. 2004;109:433-438.
2. Davis AA, Kaklamani VG. Metabolic syndrome and triple-negative breast cancer: a new paradigm. Int J Breast Cancer. 2012;2012: 809291.

3. Doyle SL, Donohoe CL, Lysaght J, Reynolds JV. Visceral obesity, metabolic syndrome, insulin resistance and cancer. Proc Nutr Soc. 2012;71:181-189.

4. Pevet P, Challet E. Melatonin: both master clock output and internal time-giver in the circadian clocks network. J Physiol Paris. 2011;105: 170-182.

5. Grossman E, Laudon M, Zisapel N. Effect of melatonin on nocturnal blood pressure: meta-analysis of randomized controlled trials. Vasc Health Risk Manag. 2011;7:577-584.

6. Peschke E, Bach AG, Mühlbauer E. Parallel signaling pathways of melatonin in the pancreatic beta-cell. J Pineal Res. 2006;40:184-191.

7. Ríos-Lugo MJ, Cano P, Jiménez-Ortega V, et al. Melatonin effect on plasma adiponectin, leptin, insulin, glucose, triglycerides and cholesterol in normal and high fat-fed rats. J Pineal Res. 2010;49:342-348.

8. Nduhirabandi F, du Toit EF, Lochner A. Melatonin and the metabolic syndrome: a tool for effective therapy in obesity-associated abnormalities? Acta Physiol (Oxf). 2012;205:209-223.

9. Koziróg M, Poliwczak AR, Duchnowicz P, Koter-Michalak M, Sikora J, Broncel M. Melatonin treatment improves blood pressure, lipid profile, and parameters of oxidative stress in patients with metabolic syndrome. J Pineal Res. 2011;50:261-266.

10. Schernhammer ES, Kroenke CH, Dowsett M, Folkerd E, Hankinson SE. Urinary 6-sulfatoxymelatonin levels and their correlations with lifestyle factors and steroid hormone levels. J Pineal Res. 2006;40:116-124.

11. Louis TA, Lavori PW, Bailar JC 3rd, Polansky M. Crossover and self-controlled designs in clinical research. $N$ Engl J Med. 1984;310: 24-31.

12. Lusardi P, Piazza E, Fogari R. Cardiovascular effects of melatonin in hypertensive patients well controlled by nifedipine: a 24-hour study. Br J Clin Pharmacol. 2000;49:423-427.

13. Brzezinski A, Vangel MG, Wurtman RJ, et al. Effects of exogenous melatonin on sleep: a meta-analysis. Sleep Med Rev. 2005; 9:41-50.

14. Buscemi N, Vandermeer B, Hooton N, et al. Efficacy and safety of exogenous melatonin for secondary sleep disorders and sleep disorders accompanying sleep restriction: meta-analysis. BMJ. 2006;332: 385-393.

15. Baumgart P, Kamp J. Accuracy of the SpaceLabs Medical 90217 ambulatory blood pressure monitor. Blood Press Monit. 1998;3: 303-307.

16. Centers for Disease Control. NHANES 2003-2004 Documentation. Available from: http://www.cdc.gov/nchs/data/nhanes/nhanes_03_04/ BM.pdf. Accessed 29 January, 2013.

17. Graham C, Cook MR, Kavet R, Sastre A, Smith DK. Prediction of nocturnal plasma melatonin from morning urinary measures. $J$ Pineal Res. 1998;24:230-238.

18. Bliwise DL, Ansari FP, Straight LB, Parker KP. Age changes in timing and 24-hour distribution of self-reported sleep. Am J Geriatr Psychiatry. 2005;13:1077-1082.

19. Buysse DJ, Reynolds CF 3rd, Monk TH, Berman SR, Kupfer DJ. The Pittsburgh Sleep Quality Index: a new instrument for psychiatric practice and research. Psychiatry Res. 1989;28:193-213.

20. Cuspidi C, Meani S, Fusi V, et al. Metabolic syndrome and target organ damage in untreated essential hypertensives. J Hypertens. 2004;22: 1991-1998.

21. Desai MY, Dalal D, Santos RD, Carvalho JA, Nasir K, Blumenthal RS. Association of body mass index, metabolic syndrome, and leukocyte count. Am J Cardiol. 2006;97:835-838.

22. Duggirala R, Blangero J, Almasy L, et al. A major locus for fasting insulin concentrations and insulin resistance on chromosome $6 \mathrm{q}$ with strong pleiotropic effects on obesity-related phenotypes in nondiabetic Mexican Americans. Am J Hum Genet. 2001;68:1149-1164. 
Open Access Journal of Clinical Trials

Dovepress

\section{Publish your work in this journal}

The Open Access Journal of Clinical Trials is an international, peerreviewed, open access journal publishing original research, reports, editorials, reviews and commentaries on all aspects of clinical tria design, management, legal, ethical and regulatory issues, case record form design, data collection, quality assurance and data auditing methodologies. The manuscript management system is completely online and includes a very quick and fair peer-review system, which is all easy to use. Visit http://www.dovepress.com/testimonials.php to read real quotes from published authors.

Submit your manuscript here: http://www.dovepress.com/open-access-journal-of-clinical-trials-journal 